Nota científica

(Short communication)

\title{
HORMIGAS (HYMENOPTERA: FORMICIDAE) ENCONTRADAS EN TRES ORQUÍDEAS (ORCHIDACEAE) DE MIQUIHUANA, TAMAULIPAS, MÉXICO
}

\section{ANTS (HYMENOPTERA: FORMICIDAE) IN THREE SPECIES OF ORCHIDS (ORCHIDACEAE) IN MIQUIHUANA, TAMAULIPAS, MEXICO}

\section{TaNia HERNÁNDEZ-LÓPEZ, ${ }^{1}$ Juana María CORONADO-BLANCO, ${ }^{1, *}$ DMitry AleXandrovich DUBOVIKOFF, ${ }^{2}$ ENRIQUE RUIZ-CANCINO ${ }^{1}$ Y JACINTO TREVIÑO-CARREÓN ${ }^{1}$}

\author{
${ }^{1}$ Facultad de Ingeniería y Ciencias, Universidad Autónoma de Tamaulipas, Centro Universitario Adolfo López \\ Mateos 87149, Cd. Victoria, Tamaulipas. \\ ${ }^{2}$ Saint Petersburg State University, Faculty of Biology, Department of Applied Ecology, 16-line of Vasiljievskij \\ Island, 29, fl 5, St. Petersburg 199178, Russia. \\ * Autora de correspondencia: <jmcoronado@docentes.uat.edu.mx>.
}

Recibido: 10/10/2016; aceptado: 19/04/2017

Editor responsable: Pedro Reyes Castillo.

Hernández-López, T., Coronado-Blanco, J. M., Dubovikoff, D. A., Ruiz-Cancino E. y Treviño-Carreón J. (2017). Hormigas (Hymenoptera: Formicidae) encontradas en tres orquídeas (Orchidaceae) de Miquihuana, Tamaulipas, México. Acta Zoológica Mexicana (n.s.), 33(2), 416-418.

RESUMEN. Tres especies de hormigas: Formica argentea Wheeler, Liometopum apiculatum Mayr, y Pseudomyrmex elongatulus (Dalla Torre) fueron observadas en orquídeas en Miquihuana, Tamaulipas, México. Además, F. argentea Wheeler se registra por primera vez para el estado de Tamaulipas.

En el estado de Tamaulipas se reportan 179 especies de hormigas (Coronado-Blanco et al., 2013; Rodríguez et al., 2014; Ríos-Cruz et al., 2015) pero en pocos casos se han registrado las hormigas y sus asociaciones con especies vegetales como es el caso del mango Mangifera indica L. (Flores \& González, 2005), de la guapilla Hechtia glomerata Zucc. (Ríos-Cruz et al., 2015) y de la palma Chamaedorea radicalis Mart. (Lara-Villalón et al., 2015). Además, no existe un registro completo de las localidades de colecta de hormigas en el estado, excepto para el municipio de Bustamante (Flores, 2012) y de Victoria (Rosas et al., 2008). Por lo tanto, el objetivo del presente trabajo fue colectar hormigas sobre orquídeas en tres localidades de Miquihuana, Tamaulipas durante agosto del 2016.

El material fue colectado manualmente de las flores
Hernández-López, T., Coronado-Blanco, J. M., Dubovikoff, D. A., Ruiz-Cancino E., \& Treviño-Carreón J. (2017). Ants (Hymenoptera: Formicidae) in three species of orchids (Orchidaceae) in Miquihuana, Tamaulipas, Mexico. Acta Zoológica Mexicana (n.s.), 33(2), 416-418.

ABSTRACT: Three ant species: Formica argentea Wheeler, Liometopum apiculatum Mayr, and Pseudomyrmex elongatulus (Dalla Torre) were observed on orchids in Miquihuana, Tamaulipas, Mexico. Moreover, F. argentea Wheeler is recorded for the first time for the State of Tamaulipas.

y raíces de orquídeas sanas (sin hemípteros) en tres localidades del municipio de Miquihuana en un gradiente altitudinal entre los 2,117 a los 2,790 msnm y diferentes tipos de vegetación [bosque de encino (Quercus miquihuanensis Nixon \& Mueller) y bosque de pinos (Pinus pseudostrobus Lindl. y P. cembroides Zucc. asociado a Agave gentryi Ullrich)]. Los especímenes fueron colocados en frascos plásticos con alcohol al 70\% y los respectivos datos de colecta. El material se encuentra depositado en el Museo de Insectos de la Facultad de Ingeniería y Ciencias de la Universidad Autónoma de Tamaulipas, en Cd. Victoria, Tamaulipas, México.

Se colectaron nueve especímenes de hormigas pertenecientes a tres especies: Formica argentea Wheeler, Liometopum apiculatum Mayr y Pseudomyrmex elonga- 
tulus (Dalla Torre), en tres especies de orquídeas: flores de Habenaria limosa (Lindl.) Hemsl. y Ponthieva schaffneri (Rchb.f.) E.W. Greenw., así como en raíces de Dichromanthus cinnabarinus (Lex.) Garay. A continuación, se cita el material examinado:

Formica argentea Wheeler, 1902: "México, Tamaulipas, Miquihuana, camino a las cabañas, bosque de Pinus pseudostrobus, 2,905 msnm, en flores de Habenaria limosa, 04-VIII-2016, col. Tania Hernández López”, tres especímenes (obreras).

Distribución en MÉxico: Baja California y Chihuahua (Vásquez-Bolaños, 2011). El género Formica se había registrado para el estado de Tamaulipas sin especies (Coronado-Blanco et al., 2013), por lo que constituye un nuevo registro para Tamaulipas.

Liometopum apiculatum Mayr, 1870: "México, Таmaulipas, Miquihuana, camino a las cabañas, bosque de encinos, 2,790 msnm, en flores de Ponthieva schaffneri, 04-VIII-2016, col. Tania López Hernández”, cuatro especímenes (obreras).

Distribución en México: Chihuahua, Coahuila, Colima, Distrito Federal, Durango, Guanajuato, Guerrero, Hidalgo, Jalisco, México, Michoacán, Morelos, Nayarit, Nuevo León, Oaxaca, Puebla, Querétaro, Quintana Roo, San Luis Potosí, Sinaloa, Sonora, Tabasco, Tamaulipas, Tlaxcala, Veracruz y Yucatán (Vásquez-Bolaños, 2011). Distribución en Tamaulipas: Gómez Farías (Rodríguez et al., 2014), Miquihuana (Dubovikoff et al., 2013).

Pseudomyrmex elongatulus (Dalla Torre, 1892): "México, Tamaulipas, Miquihuana, camino al Estanque de los Walle, 2,117 msnm, bosque de Pinus cembroides y Agave gentryi, en raíces de Dichromanthus cinnabarinus, 05-VIII-2016, col. Tania Hernández López”, dos especímenes (obreras).

Distribución en México: Jalisco, Nayarit, San Luis Potosí, Tabasco, Veracruz (Vásquez-Bolaños, 2011); Tamaulipas (sin localidad - Coronado et al., 2012; Coronado-Blanco et al., 2013).

Otras especies de hormigas se han registrado asociadas a orquídeas, Formica lemani Bondroit con Chamorchis alpina Rich. en Suiza mientras que $F$. fusca Linnaeus y $F$. rufibarbis Fabricius están asociadas a Epipactis palustris (L.) Crantz en Suecia y F. polyctena Foerster a la misma orquídea en Polonia (Vega \& Gómez, 2014). En Colombia, Ramírez et al. (2001) registraron 352 interacciones que involucran 91 especies vegetales (incluyendo orquídeas), hormigas de 67 especies y hemípteros pertenecientes a nueve familias. De Vega et al. (2014) demostraron que los volátiles florales juegan un papel importante en la polinización especializada por hormigas, su estudio incluye a Formica argentea en Polygonum cascadense W.H. Baker (Polygonaceae) y otras especies de Formica polinizando flores de orquídeas mientras que Ramírez y colaboradores (2001) indican que las raíces de plantas epífitas son un importante recurso para el anidamiento de doce especies de hormigas; otras especies forrajean o buscan refugio en este sustrato. Por su parte, en México, se ha registrado la interacción entre 15 especies de hormigas y cinco especies de orquídeas en la región del Soconusco, Chiapas (Damon \& Pérez-Soriano, 2005), las cuales son diferentes a las registradas para Miquihuana, Tamaulipas; en el presente estudio no se reporta interacción de especies, sólo es una contribución al conocimiento de las hormigas encontradas en orquídeas en el municipio de Miquihuana, además de registrar por primera vez la presencia de Formica argentea en la entidad. Actualmente se conocen 180 especies de hormigas para el estado de Tamaulipas.

AGRADECIMIENTOS. A la Universidad Autónoma de Tamaulipas por el financiamiento otorgado al proyecto "Conservación de los ecosistemas de montaña del altiplano de Tamaulipas: flora, vegetación y ornitofauna” con clave PFI2016-EB-021 y al proyecto PRODEP “Estudios taxonómicos y biológicos de plagas y enemigos naturales en México" por el apoyo para la estancia de Dmitry A. Dubovikoff en la UAT y para el trabajo de campo.

\section{LITERATURA CITADA}

Coronado-Blanco, J. M., Dubovikoff, D. A., Ruiz-Cancino, E., Vásquez-Bolaños, M., Flores-Maldonado, K. Y. \& Horta-Vega, J. V. (2013). Formicidae (Hymenoptera) del estado de Tamaulipas, México. Ciencia UAT, 7, 12-17.

Coronado B., J. M., Dubovikoff, D. A., Ruiz C., E. \& Vásquez B., M. (2012). Formicidae (Hymenoptera) en el Museo de insectos (MIFA) de la Universidad Autónoma de Tamaulipas, México. Pp. 190-200. In: E. Ruiz C. y J.M. Coronado B. (Coords.). Recursos Naturales. Libro electrónico. Fomento Editorial de la Universidad Autónoma de Tamaulipas. Cd. Victoria, Tamaulipas, México.

Damon, A. \& Pérez-Soriano, M. A. (2005). Interaction between ants and orchids in the Soconusco region, Chiapas, México. Entomotropica, 20, 59-65.

De Vega C., Herrera C. M. \& Dötterl S. (2014). Floral volatiles play a key role in specialized ant pollination. Perspectives in Plant Ecology, Evolution and Systematics, 16, 32-42.

Dubovikoff, D. A., Coronado-Blanco, J. M., Treviño-Carreón, J. \& Ruiz-Cancino, E. (2013). Distribución y características de anidación de la hormiga Liometopum apiculatum Mayr, 1870 (Hymenoptera: Formicidae) en la Sierra Madre Oriental, Tamaulipas, México. Pp. 48-52. In: F. Flores A., G. Sandoval R., H. R. Sánchez 
N., M. E. E. Hoz Z. y R. Oloarte P. (Eds.) Libro de Memorias. $24^{\circ}$ Encuentro Nacional de investigación Científica y Tecnológica del Golfo de México. Tampico, Tamaulipas, México.

Flores M., K. Y. (2012). Hormigas de la zona semiárida de Bustamante, Tamaulipas, México. Pp. 201-207. In: E. Ruiz C. y J. M. Coronado B. (Coords.). Recursos Naturales. Libro electrónico. Fomento Editorial de la Universidad Autónoma de Tamaulipas. Cd. Victoria, Tamaulipas, México.

Flores M., K. Y. \& González H., H. (2005). La mirmecofauna en árboles de mango. Pp. 483-488. In: G. Sánchez, P. Reyes y R. Dirzo (Eds.). Historia natural de la reserva de la Biosfera El Cielo, Tamaulipas, México, Universidad Autónoma de Tamaulipas, México.

Lara-Villalón M., Rosas-Mejía, M., Rojas-Fernández P. \& ReyesCastillo P. (2015). Hormigas (Hymenoptera: Formicidae) asociadas a palma camedor (Chamaedorea radicalis Mart.) en el bosque tropical, Gómez Farías, Tamaulipas, México. Acta Zoológica Mexicana (n.s.), 31, 270-274.

Ramírez M., Chacón de U., P., Armbrecht I. \& Calle Z. (2001). Contribución al conocimiento de las interacciones entre plantas, hormigas y homópteros en bosques secos de Colombia. Caldasia, 23, 523-536.

Rodríguez de L., I. R., Flores M., K. Y., Vásquez B., M. \& Horta V., J. V. (2014). Lista de especies de hormigas (Hymenoptera: Formicidae) de Tamaulipas, México. Pp. 155-165. In: A. Correa S., J.V. Horta V., J. García J. y L. Barrientos L. (Eds.). Biodiversidad Tamaulipeca. Volumen 2, Número 2. Tecnológico Nacional de México - Instituto Tecnológico de Ciudad Victoria, Tamaulipas, México.

Rosas, M., Horta V., J. V., Flores M., K. Y. \& Correa S., A. (2008). Formicidae de la zona urbana de Ciudad Victoria, Tamaulipas, México. Entomología Mexicana, 7, 804-807.

Ríos-Cruz, E., Coronado-Blanco J. M., Vásquez-Bolaños M. \& Treviño-Carreón J. (2015). Camponotus senex (Smith, 1858) (Hymenoptera: Formicidae): nuevo registro para Tamaulipas. Dugesiana, 22, 63-64.

Vásquez-Bolaños, M. (2011). Lista de especies de hormigas (Hymenoptera: Formicidae) para México. Dugesiana, 18, 95-133.

Vega, C. de \& Gómez J. M. (2014). Polinización por hormigas: conceptos, evidencias y futuras direcciones. Ecosistemas, 23, 48-57. 